\title{
EL TRABAJADOR UNIVERSITARIO: ENTRE EL MALESTAR Y LA LUCHA
}

\author{
Silvia Tamez González \\ Josué Federico Pérez Domínguez
}

\begin{abstract}
RESUMEN: Las modificaciones en el mundo del trabajo producidas por la crisis de acumulación capitalista han transformado así mismo las condiciones de desarrollo de la labor docente. En un mundo cada vez más complejo y competitivo, la institución universitaria es atravesada por múltiples y contradictorias exigencias que se manifiestan en la salud de sus trabajadores. Este trabajo analiza fenómenos como la precarización del trabajo docente y sus implicaciones en la salud física y mental de los trabajadores universitarios, revisando las investigaciones recientes sobre el síndrome de burnout y padecimientos similares en México. Así mismo reflexiona sobre los límites y posibilidades de la acción política emancipadora por parte de los trabajadores de la educación.
\end{abstract}

Palabras clave: Malestar docente. Burnout. Precarización. Sindicalismo universitario.

\section{Professors: BETWEEN DISCOMFORT AND FIGHT}

ABSTRACT: The crisis of capitalist accumulation has brought changes in the world of work that have also affected the working conditions of professors. In a world increasingly complex and competitive, universities are traversed by multiple and conflicting demands that have impacts on their workers' health. This paper analyzes the phenomena of precarious work in the teaching profession and its implications for the physical and mental health of university

* Profesora-investigadora del Área Estado y Servicios de Salud, Universidad Autónoma Metropolitana (UnAm, México).E-mail: stamez@correo.xoc.unam.mx

** Ayudante de investigación del Área Estado y Servicios de Salud (unam, México). E-mail: josuefpd@gmail.com

Educ. Soc., Campinas, vol 30, n. 107, p. 373-387, maio/ago. 2009

Disponível em <http://www.cedes.unicamp.br> 
employees. It reviews recent research on the burnout syndrome and similar sufferings in Mexico to reflect on the limits and possibilities of emancipatory political action by education workers.

Key words: Teaching discomfort. Burnout. Precarious work. University unionism.

\section{Crisis capitalista y educación}

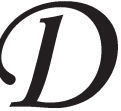

espués del crack económico de 1929-1932, los capitalistas y gobiernos del mundo comprendieron que para sobrevivir y recuperar su poderío económico necesitaban de la participación y control directo de los Estados nacionales sobre los procesos sociales y económicos generales. Desde entonces, y en el marco de lo que se llamó Estado benefactor, las masas obreras y campesinas del mundo encontraron respuestas más o menos satisfactorias a sus necesidades y exigencias, como la salud, las pensiones, la educación, la seguridad en el trabajo etc.

Desde la crisis del patrón de acumulación en 1970-1973, el capitalismo mundial ha inaugurado una nueva fase de "acumulación por desposesión” (Harvey, 2007), lo que conocemos como neoliberalismo, el cual se ha instalado mediante golpes de Estado, bombas y leyes, prácticamente en la totalidad del planeta. Diversos autores en los últimos 20 años han hablado de que el capitalismo está llegando a sus límites geográficos y políticos. Ya no hay "Américas por descubrir", ni "lejanos oestes" por conquistar (Wallerstein, 1998). Por tal motivo, el neoliberalismo, según Harvey, recurre a estrategias parecidas a lo que Marx llamó "acumulación originaria" de capital, es decir, al despojo de los bienes públicos de importantes sectores sociales, tales como las tierras comunales, los ahorros y pensiones de los trabajadores de las ciudades o los sistemas públicos de educación y salud (Harvey, 2007). En el ámbito laboral se ha pretendido debilitar a los sindicatos e instituciones públicas que protegen los derechos de los trabajadores, ya sea modificando las leyes, impulsando "nuevas culturas laborales" o corporativizando a las organizaciones de trabajadores.

La presente crisis mundial, la más grande en ochenta años, acelera la privatización de los bienes públicos y la conformación de los contenidos de la educación a las necesidades de un nuevo proceso de 
acumulación de capital que permitiría a los grandes capitalistas salir del atolladero. De ahí el ataque a la universidad pública y su conformación neoliberal en la Unión Europea, como lo muestra el conflicto estudiantil en Bolonia, o la desaparición de la materia de filosofía en los planes de estudio del nivel medio superior en México, entre muchos otros ejemplos.

En este contexto, los procesos de trabajo han sido escenario de profundas transformaciones organizativas, que en nuestros países se traducen, sobre todo, en "flexibilización" de la fuerza de trabajo, acompañada de algunos cambios tecnológicos que lejos de reducir las cargas de trabajo, vienen a exigir de parte de los trabajadores una mayor adaptabilidad y re-certificación de sus habilidades.

Por supuesto, el proceso de trabajo del docente no ha escapado a estos cambios que desde una perspectiva general, están determinados por el cambio de asignación del papel social de la educación, cuyo sentido emancipador ha perdido valor ante su nuevo objetivo: ser espacio "privilegiado" de la reproducción del capital y herramienta de la expansión capitalista.

\section{El proceso de trabajo docente: alienación y despersonalización}

La flexibilización neoliberal del trabajo impone mecanismos a través de los cuales se pretende extraer mayor plusvalía del trabajo humano vivo. Mediante políticas globales dictadas a los gobiernos desde el Banco Mundial y la Organización Mundial de Comercio, se aprueban leyes, códigos y reglamentos que permiten a las clases dominantes un mayor control sobre el proceso de trabajo. La productividad, calidad, eficiencia y competitividad, tan propulsadas en los últimos 20 años, son las nuevas cadenas invisibles con las que los grandes capitalistas imprimen al proceso de trabajo un ritmo, un rumbo y una cualidad necesarias para impulsar aún más su proyecto de explotación intensiva y acumulación infinita de capital, sin importarles la destrucción de los derechos sociales y económicos conquistados durante décadas de luchas obreras, ni el empobrecimiento de las condiciones de vida, ni la salud física y mental de las y los trabajadores en general.

En cuanto al proceso de trabajo docente en México, su transformación se empieza a hacer evidente en la primera mitad de los años 
noventa con las reformas al artículo $3^{\circ}$ constitucional y los sucesivos programas neoliberales de educación instrumentados por los gobiernos del PRI y el PAN en las últimas décadas.

El trabajador docente se aliena de sí mismo porque pierde cada vez más el control sobre su propio proceso de trabajo y sobre el producto de su trabajo. El proceso de globalización neoliberal imprime al trabajo docente nuevas exigencias y necesidades acordes con la función social que los gobiernos esperan de la universidad pública. "A ésta... ahora se le asigna la responsabilidad de reactivar a la industria, de ofrecerle soluciones tecnológicas creativas y las transferencias que garanticen los nichos de competitividad para la industria de nuestro país" (Aboites, 1994, p. 46).

Esta idea de la universidad como proveedora de mano de obra altamente calificada y productora de conocimientos orientados a satisfacer las necesidades de expansión de las grandes empresas tiene consecuencias desastrosas en todos los ámbitos de la universidad. En lo que respecta al trabajo docente, decíamos, se pierde la capacidad de controlar el propio proceso y los objetivos de la docencia, lo que tiene como consecuencia, entre otras muchas, la despersonalización de las relaciones entre docente y alumnos, y entre colegas docentes.

La despersonalización de las relaciones humanas no es algo solamente propio del mundo del trabajo, en este caso docente; es propio de todo el entramado de relaciones sociales en el capitalismo. Como decía Marx (1986, p. 38), las relaciones sociales aparecen "no como relaciones directamente sociales de las personas en sus trabajos, sino como relaciones materiales entre personas y relaciones sociales entre cosas".

En el aula, el profesor(a) aparece ante los estudiantes como el agente de autoridad que los disciplina, escolariza y evalúa, y ante el cual establecen estrategias de adaptación para lograr el objetivo de aprobar su materia. Los alumnos son para el profesor(a) la masa amorfa, más que con nombre y apellidos, con número de matrícula, a la que hay que moldear con la fuerza de su saber, para así cumplir con la cuota de horasaula necesarias para permanecer como docente-investigador de excelencia dentro de los criterios productivistas de la institución educativa.

El proceso de enseñanza-aprendizaje, que debiera ser como un manantial dialógico en el que tanto alumnos como docente se encuentran como humanos y comparten sus saberes y experiencias, se 
convierte mediante la despersonalización de sus relaciones, en un trámite burocrático, en el que los ocultamientos y las apariencias mutuas tienen como objetivo utilitario cumplir con el compromiso socialmente (des)valorado de "estar en la Universidad".

De la misma manera, el producto del trabajo de investigación (artículos, ponencias, modelos etc.), dependiendo de la especialización de cada área de investigación, se convierte la mayoría de las veces en un "objeto ajeno" de su productor. El investigador es un trabajador de la "maquila del conocimiento" (Subcomandante Insurgente Marcos, 2006), produce una pequeña parte del conocimiento científico y desconoce el producto final; la totalidad del proceso del conocimiento científico y su aplicación práctica y/o tecnológica, generalmente le es ajena.

Si a esto agregamos que para certificar todas sus actividades, los docentes tienen que cumplir con una serie de trámites administrativos, las más de las veces absurdos y exhaustivos, la "jaula invisible" de la enajenación burocrática termina cerrándose sobre su persona. Como dice Crabay (2001, p. 6): "Los altos mecanismos burocráticos los convierten en un autómata que llena papeles e integra comisiones generándose así un movimiento que lo aliena y lo vuelve ajeno a sí mismo y a sus producciones".

Aunque las leyes orgánicas de las universidades públicas del país establecen su carácter de "autónomas", y por lo tanto, los mecanismos de evaluación de sus académicos, supuestamente, son establecidos y aplicados por los propios académicos de cada institución, los criterios que se han ido introduciendo para controlar y evaluar dicho trabajo son una clara respuesta a los parámetros que los gobiernos neoliberales imponen a las instituciones de educación superior para otorgarles o retirarles presupuesto de acuerdo a su productividad y su "contribución al Plan Nacional de Desarrollo", como es el caso del Sistema Nacional de Investigadores (SNI).

Así mismo, con la creación de instancias certificadoras ajenas a las propias universidades se pretende estandarizar los contenidos y objetivos de los planes y programas de estudio de licenciaturas y posgrados, para adaptarlos a las necesidades del "conocimiento global" de las empresas trasnacionales.

El trabajo universitario deja de considerarse desde una perspectiva intrainstitucional, para verse ahora más bien desde fuera. De ahí se desprende 
la tendencia a considerar el trabajo universitario como necesariamente sujeto a una evaluación en la cual debe participar su demandante fundamental, es decir, el sector empresarial de la globalización económica (...). (Aboites, 1994, p. 47)

Ésta condicionada "autonomía” se ve aún más restringida en el caso mexicano si consideramos que los empresarios tienen una injerencia real en el proyecto educativo nacional al menos en dos aspectos. Primero, al formar parte del Consejo para la Formación de Recursos Humanos en Ciencia y Tecnología del Consejo Nacional de Ciencia y Tecnología (CONACYT), que es la institución del Estado mexicano que evalúa, financia y dirige los más importantes proyectos de investigación y desarrollo científico y tecnológico en el país. Segundo, los gobiernos neoliberales de los últimos 20 años han impulsado la creación de institución de educación superior, llamadas universidades tecnológicas, en las cuales los empresarios de distintas ramas productivas son miembros del consejo directivo y diseñadores directos del currículum académico de las mismas. Y el más reciente caso, el intento de aprobación del proyecto de crear una nueva unidad de la Universidad Autónoma Metropolitana, la Unidad Lerma, totalmente subordinada al sector empresarial que pretende crear una "ciudad del conocimiento" en el municipio de Lerma de Villada, en el Estado de México (con intensa actividad agropecuaria e industrial), que le suministre la mano de obra altamente especializada en ciencias y tecnologías de punta (como la nanotecnología, la genómica etc.) y las innovaciones científico-tecnológicas que apuntalen la competitividad y la productividad.

Con el discurso de la calidad y la excelencia en la educación no han logrado convencer ni a los estudiantes, cada vez más excluidos de las instituciones de educación pública, ni a los académicos, cada vez más mermados en su calidad de vida por "la reducción de los salarios, los pagos a destajo y la escasez y métodos de entrega de recursos para la instituciones". Lejos de haber mejorado la calidad y extensión de la educación en México, los gobiernos neoliberales se han encargado de avanzar en la privatización de las universidades públicas y en tecnificar la enseñanza en los niveles básico, medio-superior, y ahora superior. De esta manera, para los académicos "la calidad y excelencia se convierten simplemente en una superimposición de trámites y rituales sobre una realidad antigua de feudos disciplinarios y políticos que no ha sido 
cambiada o sobre un taylorismo que produce ponencias como en línea de montaje" (Aboites, 1994, p. 48).

Así, es que si bien existen un grupo importante de profesores que reciben y disfrutan de becas, estímulos y premios otorgados como recompensas a someter más su tiempo y energía a los cánones productivistas, cada vez más, las necesidades del trabajo docente se van llenando con académicos contratados por horas o por medios tiempos, que no pueden aspirar a obtener estos estímulos y que son contratados en condiciones de precariedad laboral. Estos docentes tienen que esperar pacientemente a que uno de los "profesores viejos" se retire o muera para aspirar al disfrute de una vida digna y a desarrollarse profesional y personalmente de manera plena. Estos trabajadores precarios de la labor docente representan un alto porcentaje, alrededor de 60\% según algunas estimaciones, (Cuninghame, 2008), del total de trabajadores dedicados a la educación. Tienen que sobrevivir yendo de una escuela a otra, de un grupo a otro, produciendo y enviando artículos a revistas y simposios para incrementar su status de productividad y su experiencia laboral. Sobre sus hombros y desvelos es que se sostienen los "expertos" evaluadores y diseñadores de programas de alta "calidad" y "eficiencia".

Y no sólo eso, además de establecer arbitraria e injustamente cada vez más categorías y estratos dentro del sector académico para así ir filtrando a quienes tienen y no derecho a acceder al presupuesto, las consecuencias que éstas medidas y trámites burocráticos de evaluación y control del trabajo docente tienen en la salud mental de los trabajadores son indiferentes para los repartidores de canonjías, y pueden ir desde un simple estrés matutino hasta la destrucción de la personalidad y la psicosis (Crabay, 2001).

\section{La muerte civil y política del trabajador docente}

La conciencia de clase de los trabajadores no es algo que se da de manera inmediata, no está dada por la simple posición que se ocupe en las relaciones sociales de producción. La clase, así como su conciencia y organización, se constituye mediante las experiencias, triunfos, derrotas y anhelos, personales y colectivos, que la propia lucha de clases conlleva.

El "aburguesamiento" de los trabajadores académicos no se debe solamente a que sean trabajadores intelectuales y que, a diferencia de 
los trabajadores manuales, tengan otras necesidades e intereses; sino que se debe también a las experiencias de frustración política que las recientes derrotas sindicales han impreso en su conciencia, así como a que los dispositivos de dominación económica e ideológica instrumentados por las clases dominantes han sido efectivos y más o menos exitosos.

La dominación ideológica del capital tiene en la fragmentación de las clases subalternas una de sus características más eficientes (Thompson, 1987). Hacerles creer a los trabajadores docentes que si trabajan más y de acuerdo a los parámetros y criterios que los agentes intelectuales del capital establecen, van a ganar más, vivir mejor y proyectarse profesionalmente a más alto nivel, los despoja de cualidades humanas como la solidaridad y la responsabilidad con los otros, así como de la creación colectiva y fraterna del conocimiento y su socialización no mercantilizada.

A ello se debe precisamente que los trabajadores académicos estén más preocupados por mantener su estatus social, su nivel de consumo y su seguridad personal y familiar, que por participar en las reformas necesarias de la universidad, en la defensa de la educación pública o en la transformación profunda de la sociedad.

La universidad como espacio libre, de análisis crítico y elaboración de propuestas de solución a los problemas de nuestra época; la universidad como proyecto humanizador y emancipatorio, queda de esta manera reducida a un ámbito cada vez más cerrado de competencia mercantil y compadrazgo político.

Si bien es cierto que, como advertía Marx, en los momentos en que el sistema político-económico se ve seriamente amenazado por las crisis propias del capitalismo y por el empuje de las fuerzas progresistas de la sociedad, las clases medias se ven obligadas a definirse entre estar con los de "arriba" o estar con los de "abajo". La actual situación de anomia, perplejidad, apatía e incapacidad de organización y solidaridad de los trabajadores académicos universitarios los pone en la riesgosa y vergonzante condición de "víctimas" pasivas que padecen el proceso de globalización, en lugar de participar y conducirlo activa y conscientemente.

\section{La salud del trabajador docente}

Ante este panorama cabe preguntarse: ¡es la educación superior un terreno conflictivo generador de abundantes factores estresantes y 
potencialmente patológicos? La respuesta es sí, porque a pesar del intento transgeneracional de hacer de la universidad "una esfera pública de vital importancia para desarrollar y nutrir el balance apropiado entre las esferas públicas y democráticas y el poder comercial" (Giroux, 2002 , p. 12), lo que ocurre en realidad es que el poder de las mercancías termina por inclinar la balanza a su favor. Así como la universidad es un campo generador de creatividad y conocimientos valiosos para la sociedad, también es un campo en donde se dan cita todas sus fuerzas sociales y políticas. Saber adaptarse a esa conflictividad y combatir dentro de sus marcos legales y culturales, no es algo para lo que ésta completamente preparado el trabajador docente. Las habilidades teóricas y prácticas con las que cuenta, se ven constantemente desvaloradas y sometidas a escrutinio y desarticulación.

Las cargas de trabajo académico con las que tienen que lidiar diariamente los docentes muchas veces sobrepasan su capacidad de resistencia física y psíquica. Es sabido que una de las principales causas de malestar en los docentes se debe a un ambiente de trabajo que perciben como hostil y altamente competitivo. En estas circunstancias la hipocresía entre colegas no basta para permitir el desarrollo creativo de sus potencialidades, por lo que ante el "quiebre de las relaciones interpersonales" (Crabay, 2001, p. 8), los docentes reaccionan de muy distintas formas, desde la negación de estar "quemados" hasta el refugio reactivo en la religión o el consumo de alcohol u otras drogas.

Numerosos estudios han categorizado y explicado las distintas fases sintomáticas del síndrome de estar "quemado" y han recomendado a los trabajadores de la salud y la educación estar pendientes para identificarlo. Pero la patología laboral del trabajador docente no se limita solamente al denominado burnout y a los aspectos psicosociales. La cantidad de riesgos a los que se exponen los docentes son múltiples y pueden contarse entre ellos también, problemas posturales, uso excesivo de la voz, toda la gama de problemas derivados del uso intensivo de computadora, problemas circulatorios, exposición a agentes físicos y químicos etc.

A continuación se presenta una breve revisión de estudios realizados en México en personal docente, en los que se exploran algunos de estos problemas de salud pero de manera especial el sindrome de burnout. 


\section{Evidencias empíricas en México}

Podemos considerar que el interés por este tema en nuestro país es reciente y no existen muchos estudios que incorporen el proceso de trabajo universitario desde una perspectiva amplia e integral. Así mismo, en una revisión rápida de investigaciones realizadas encontramos que la mayoría de ellos se dirigen fundamentalmente a la patología laboral tipificada por la Ley Federal del Trabajo para los trabajadores docentes, ocupando un lugar preponderante la laringitis por uso intensivo de la voz y la neurosis. Por lo general, estas investigaciones hacen referencia a los profesores de primaria y secundaria. Sin embargo, específicamente en lo que tiene que ver con el trabajo universitario, encontramos algunos hallazgos de importancia.

Por ejemplo, Manuel Pando y Carolina Anda (Pando \& Anda, 2003 y 2006), desde una perspectiva de psicología organizativa, han estudiado la prevalencia de síndrome de burnout en estudiantes de posgrado y docentes y mobbing solamente en docentes universitarios. Según sus resultados no hay evidencia de que la alta prevalencia de mobbing $(79,7 \%)$ en docentes universitarios esté fuertemente relacionada con el "sistema de trabajo". Lo curioso de esta investigación es que, a pesar de que en sus conclusiones nos conminan a tomar sus resultados con las "precauciones de todo estudio inicial", proponen "cambios culturales a nivel de los valores, actitudes, expresiones verbales y modos de interacción del individuo", así como "cambios en la cultura organizacional de los centros de trabajo" (p. 199). Es decir, reducen el problema a medidas puntuales propias de un abordaje gerencial de la salud laboral.

Por su parte, Roberto Baltazar Montes (2001) realizó un interesante estudio-taller con educadores de personas jóvenes y adultas para medir el nivel y la capacidad de percepción del malestar docente. Los resultados arrojados por este estudio-taller muestran que la más del $50 \%$ de los participantes no sabían del síndrome de burnout ni del malestar docente, pero a través de la escucha y comunicación reflexiva pudieron identificar problemas de salud comunes, así como plantear exigencias de disminución de carga de trabajo y necesidades de reconocimiento moral y económico mayores. La verbalización de su malestar permitió a los docentes, según el coordinador-autor del estudio-taller, revalorar su labor como educadores y la importancia "de saber escuchar su cuerpo". 
Llaman la atención dos investigaciones en las que se recupera una visión más amplia e integradora del problema arriba mencionado. Uno de los trabajos realizado por Arturo Juárez García (2004), de la Universidad Nacional Autónoma de México, desarrolló un original cuestionario de control personal, con variables recién validadas como "control emocional" y "autoeficiencia", que le permitió realizar un estudio en Organizaciones de Servicio Humano (con personal de enfermería, psicología, medicina, trabajo social, educación y vigilancia), que lo llevó a la conclusión de que "la inseguridad laboral fue el estresor más nocivo para la salud psicológica” (p. 194).

Al contrario de lo postulado por la conocida y ampliamente usada propuesta de Karasek, este estudio revela que

(...) el contexto socioeconómico y cultural influye en la nocividad de ciertas variables, pues aunque el modelo mencionado considera que sus variables son "universales", en lo que refiere a la salud mental en este tipo de trabajadores mexicanos, parece que son más relevantes otros aspectos propios de nuestra historia y devenir actual, como lo es la gran incertidumbre en la estabilidad de los empleos y la inseguridad provocada por la situación socioeconómica y política por la que atraviesa nuestro país. (p. 194)

Estas conclusiones son importantes pues promueven una reflexión teórica y metodológica sobre el uso de modelos desarrollados por investigadores de otros países con menos desigualdad social que los nuestros y que, con frecuencia, llevan a subvalorar la importancia de elementos que conforman la situación económica y social que son determinantes del proceso salud enfermedad.

Otra investigación de más antigua data, realizada por Margarita Vázquez Fernández sobre los "Daños a la salud asociados a los programas de estímulos en académicos del Colegio de Postgraduados en Ciencias Agrícolas", en 1999, en la que se incorpora el proceso de trabajo desde una perspectiva amplia, se concluye que los profesores que pertenecen al Sistema Nacional de Investigadores y a los programas de evaluación de becas y estímulos manifestaron padecer más "'trastornos mentales' (irritabilidad, ansiedad, depresión, trastornos del sueño)... [así como] otras enfermedades, tales como insuficiencia vascular periférica (várices y hemorroides)... fatiga crónica y padecimientos dorsolumbares". Ante esta evidente relación entre aumento de exigencias 
de trabajo y evaluación y deterioro de la salud, convendría preguntarse si "desde el punto de vista ético y de estricta justicia laboral ¡cabe exigir un costo tan elevado de salud, relaciones familiares, calidad de vida y decesos prematuros a cambio de un aumento discutible o muy probablemente marginal en la productividad científica nacional?" (Aboites, 2001).

Sin embargo, una investigación reciente, en la que se estudió al conjunto de los trabajadores universitarios, específicamente en la indagación de la prevalencia de riesgo de enfermedad cardiovascular como una especie de "trazador" de exposición a estrés, muestra interesantes resultados. El estudio (Becerril, 2008) se realizó en trabajadores de la Universidad Autónoma Metropolitana (Xochimilco, México) y se incluyó a todos los trabajadores de base. Dado que las actividades laborales son muy diferentes de acuerdo al tipo de trabajo realizado, se comparó la prevalencia de riesgo cardiovascular entre docentes, administrativos y trabajadores de servicios generales.

Los resultados mostraron que los docentes presentan las mejores condiciones laborales pues califican su trabajo como de alta demanda, pero con mucho control y con apoyo social por parte de compañeros y jefes. También consideran que su trabajo es de alto esfuerzo y altas recompensas y con sobrecompromiso. Así mismo, son quiénes presentan menos exposición a factores psicosociales negativos, cuyo estado nutricional es adecuado y que por lo tanto tienen menos riesgo cardiovascular.

Por su parte, los trabajadores de servicios generales reportaron tener bajas demandas de trabajo, bajo nivel de control y altos niveles de conflictividad en relaciones sociales dentro del trabajo y son quienes presentan mayor prevalencia de sobrepeso, hipertensión arterial, consumo de tabaco y alcoholismo y además son los que realizan menor actividad física, lo que explica que también fueron los que registraron las tasas más altas de riesgo cardiovascular. El grupo de trabajadores administrativos fue el que mostró mayor frecuencia de obesidad.

Es decir, a pesar de que los docentes están expuestos a mayor compromiso y demanda de trabajo, cuentan con mejores condiciones generales de vida y con más "amortiguadores" ocupacionales como la satisfacción y la creatividad que involucra la actividad docente. 


\section{Conclusiones}

Los cambios operados durante los últimos 20 años en la organización del trabajo universitario han ocasionado mayor carga psicológica para los docentes. Es decir, las exigencias laborales se han diversificado en un sinnúmero de aspectos propios de la docencia y otros que responden a la necesidad de competitividad y reconocimiento en el medio académico.

Sin duda, la organización taylorista del trabajo, a partir de la introducción de estímulos a la productividad a los que puede accederse por distintas vías, ha sido uno de los principales motores de estos cambios, pues han conformado condiciones de trabajo y de vida muy diferenciadas al interior del grupo docente y también en relación con todos los trabajadores que hacen posible que las universidades cumplan con sus funciones.

La actividad del docente se ha individualizado y fragmentado, por lo que los objetivos del quehacer docente son más difícilmente compartidos y defendidos colectivamente. Reconocernos como trabajadores, y por lo tanto como sujetos sometidos a la gran maquinaria político-económica de la explotación mundial, nos permitirá entonces mirarnos en las distintas miradas del resto de los trabajadores de América y el mundo. Reconocernos no sólo como trabajadores universitarios, sino como pertenecientes a una difuminada y fragmentada clase trabajadora, nos permitirá reflexionar crítica y renovadamente sobre el rumbo que el sindicalismo universitario va tomando, impulsado por las grandes transformaciones económicas, políticas, tecnológicas y culturales de nuestra época.

Recuperar la universidad como el ámbito de lo público donde se tejen las más ricas y complejas relaciones sociales que, por tener como materia de trabajo todas las expresiones de la ciencia, el arte y la cultura, no pueden menos que mirarse como contradictorias y múltiples, al mismo tiempo sometidas a la lógica dominante del capital que propulsoras de la reflexión crítica y la transformación humanizadora del mundo.

Ese es el proyecto político que los trabajadores académicos debemos construir y defender frente a la homogenización del pensamiento y la vida propugnada por el capital trasnacional. Y no podremos llevarlo a 
cabo encerrados en nuestros cubículos, protegidos por nuestras becas y estímulos, concentrados en nuestro inmenso pedacito de saber tecnificado, escondiendo la cabeza y el cuerpo a las agresiones directas y evidentes para todos contra la universidad pública. Recuperar en nosotros la concepción y la ejecución del trabajo académico, arrebatándoselo al SNI, al Banco Mundial, a los ministros de Educación, y a todos los "expertos" que nos enajenan la riqueza de nuestro quehacer y la satisfacción de contribuir libremente a la construcción de un mundo mejor.

Recebido em maio de 2009 e aprovado em julho de 2009.

\section{Referencias}

ABOITES AGUILAR, H. El futuro de nuestra carrera académica. Revista Reencuentro, México, DC, n. 12, ago. 1994.

ABOITES AGUILAR, H. Estrés e investigación. La Jornada, México, DC, 14 mayo 2001, Lunes en la Ciencia.

BECERRIL, Y.A. Diferencias en riesgo cardiovascular en función del puesto de trabajo en la UAM Xochimilco. 2008. Tesis (Maestra en Ciencias en Salud de los Trabajadores) - Universidad Autónoma Metropolitana, Xochimilco, México, DC.

BALTAZAR MONTES, R. El malestar docente en las y los educadores de personas jóvenes y adultas, 2001. Disponível em: <www. comie.org.mx/congreso/memoria/v9/ponencias/at15/PRE1178887416. pdf>. Acesso em: jul. 2009.

CARAMÉS BALO, R. Precarización y burnout del puesto de trabajo del personal docente e investigador de la universidad. Revista Praxis, n. 2, p. 13-21, abr. 2003. Disponível em: <www.revistapraxis.cl/ ediciones/numero2/carames_praxis_2.htm>. Acesso em: jun. 2008.

CAROLINA, A.; PANDO, M. Síndrome de burnout y factores psicosociales en el trabajo, en estudiantes de postgrado del Departamento de Salud Pública de la Universidad de Guadalajara. Revista Psiquiatría Fac Med Barna, México, DC, v. 4, n. 30, p. 193-199, 2003. 
CARREÑO GARCÍA, S. et al. Características organizacionales, estrés y consumo de alcohol en trabajadores de una empresa textil mexicana. Salud Mental, México, DC, v. 29, n. 4, jul./ago. 2006.

CRABAY, M.I. Salud mental y educación superior: malestar docente. Asociación Argentina de Especialistas en Estudios del Trabajo, Argentina, 2001. Disponível em: <http://www.aset.org.ar/congresos/5/ aset/PDF/CRABAY.PDF>. Acesso em: jun. 2008.

CUNINGHAME, P. "Edufatory": precarización de la producción del conocimiento y alternativas; ponencia para Mesa 3: "Precariedad del trabajo universitario y conflicto en las instituciones de educación superior". In: Coloquio "Los Retos del Sindicalismo Universitario Frente a la Precarización del Trabajo en las Instituciones de Educación Superior" y el II Encuentro Internacional sobre Trabajo Precario, México, DF, 11-13 ago. 2008.

GIROUX, H.A. Intelectuales públicos y la política educacional. Revista Praxis, n. 1, p. 12-18, 2002.

HARVEY, D. El neoliberalismo como destrucción creativa. (Trad. of The Annals of the American Academy of Political \& Social Science, USA, 2007). Disponível em: <http://www.rebelion.org/noticia.php?id=65709>. Acesso em: jun. 2008.

JUÁREZ GARCÍA, A. Factores psicosociales relacionados con la salud mental en profesionales de servicios humanos en México. Ciencia \& Trabajo, Chile, v. 6, n. 14, p. 189-196, oct./dic. 2004.

MARX, K. El capital. México, DC: FCE, 1986. t.1.

PANDO, M.; ARANDA, C. Factores psicosociales de la organización asociados a la presencia de mobbing en docentes universitarios. Revista Psiquiatría Fac Med Barna, Barcelona, v. 33, n. 1, p. 42-47, 2006.

RIVERA RAMÍREZ, M.G.; RIVAS MEDINA, J. Salud mental universitaria. Revista Electrónica "La Misión”, México, DC, 2004. Disponivel em: <www.uaq.mx/psicologia/lamision/smuniversitaria.html>.

THOMPSON, J. Language and ideology. The Sociological Review, London, v. 35, n. 3, p. 516-536, ago. 1987.

WALLERSTEIN, I. El capitalismo histórico. 3. ed. México, DC: Siglo XXI, 1998. 